\title{
Supporting Information: DNA Looping and DNA Conformational Fluctuations Can Accelerate Protein Target Search
}

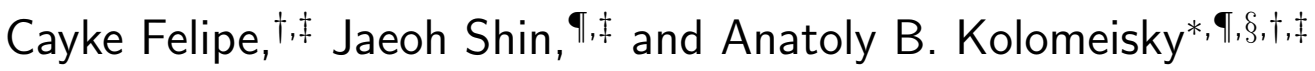 \\ $\dagger$ Department of Physics and Astronomy, Rice University, Houston, Texas 77005, USA \\ $\ddagger$ Center for Theoretical Biological Physics, Rice University, Houston, Texas 77005, USA \\ IDepartment of Chemistry, Rice University, Houston, Texas 77005, USA \\ $\S$ Department of Chemical and Biomolecular Engineering, Rice University, Houston, Texas \\ 77005, USA \\ E-mail: tolya@rice.edu
}

\section{Details of the derivation of the mean search times}

In this Supporting Information we provide the details of derivations for the target search time $T$ shown in the main text. The first-passage probability functions are governed by the backward master equations.

$$
\frac{\partial F_{n}(t)}{\partial t}=k_{\mathrm{off}} F_{0}(t)+u\left[F_{n-1}(t)+F_{n+1}(t)\right]-\left(k_{\mathrm{off}}+2 u\right) F_{n}(t)
$$


for $n=2,3,4, \ldots, L-1$ and $n \neq l$. For the boundary and other special states we have,

$$
\begin{aligned}
& \frac{\partial F_{1}(t)}{\partial t}=k_{\text {off }} F_{0}(t)+u F_{2}(t)-\left(k_{\text {off }}+u\right) F_{1}(t), \\
& \frac{\partial F_{l}(t)}{\partial t}=k_{\text {off }} F_{0}+u\left[F_{l-1}(t)+F_{l+1}(t)\right]+k_{t} F_{L}(t)-\left(k_{\text {off }}+2 u+k_{t}\right) F_{l}(t), \\
& \frac{\partial F_{L}(l)}{\partial t}=k_{\text {off }} F_{0}(t)+u F_{L-1}(t)+k_{t} F_{l}(t)-\left(k_{\text {off }}+u+k_{t}\right) F_{L}(t) .
\end{aligned}
$$

The backward master equation that describes the temporal evolution of the protein in the bulk $(n=0)$ is given by

$$
\frac{\partial F_{0}(t)}{\partial t}=k_{\mathrm{on}} \sum_{n=1}^{L} F_{n}(t)-L k_{\mathrm{on}} F_{0}(t)
$$

Finally, $F_{m}(t)=\delta(t)$, which means that if the initial position of the searching protein is already at the target site $m$ at $t=0$, the process ends immediately. We apply the Laplace transform, $\widetilde{F}_{n}(s) \equiv \int_{0}^{\infty} F_{n}(t) \exp (-s t) d t$, where $s$ is the Laplace variable. Then we have

$$
\begin{aligned}
& \left(s+2 u+k_{\text {off }}\right) \widetilde{F}_{n}=k_{\text {off }} \widetilde{F}_{0}+u\left[\widetilde{F}_{n-1}+\widetilde{F}_{n+1}\right] \\
& \left(s+u+k_{\text {off }}\right) F_{1}=k_{\text {off }} \widetilde{F}_{0}+u \widetilde{F}_{2} \\
& \left(s+2 u+k_{t}+k_{\text {off }}\right) \widetilde{F}_{l}=k_{\text {off }} \widetilde{F}_{0}+u\left[\widetilde{F}_{l-1}+\widetilde{F}_{l+1}\right]+k_{t} \widetilde{F}_{L} \\
& \left(s+u+k_{t}+k_{\text {off }}\right) \widetilde{F}_{L}=k_{\text {off }} \widetilde{F}_{0}+u \widetilde{F}_{L-1}+k_{t} \widetilde{F}_{l} \\
& \left(s+L k_{\text {on }}\right) \widetilde{F}_{0}=k_{\text {on }} \sum_{n=1}^{L} \widetilde{F}_{n} .
\end{aligned}
$$

In order to solve the above coupled difference equations, we use the ansatz $\widetilde{F}_{n}=A y^{n}+$ $B y^{-n}+C$ for $n=1,2,3, \ldots, L$, where $y \equiv \frac{\left(s+2 u+k_{\text {off }}\right)-\sqrt{\left(s+2 u+k_{\text {off }}\right)^{2}-4 u^{2}}}{2 u}$. It can be shown that that $C=\frac{k_{\text {off }}}{s+k_{\text {off }}} \widetilde{F}_{0}$. In order to solve this problem for all parameters value we need to consider two cases depending on the target site $(m)$ and the transfer site $(l)$ locations. For both cases, the DNA strand can be divided into three regions. 


\section{Transfer site located before target, $l \leq m$}

In this case, for the three regions on DNA we can write different first-passage probabilities:

- $\widetilde{F}_{n, 1}(y)$, for $1 \leq n \leq l$

- $\widetilde{F}_{n, 2}(y)$, for $l \leq n \leq m$ and

- $\widetilde{F}_{n, 3}(y)$, for $m \leq n \leq L$.

Now, as each $\widetilde{F}_{i, n}(y)$, with $i=1,2,3$, will have its own set of $\left\{A_{i}, B_{i}\right\}$ constants that can be found using the boundary conditions presented in Eqns. S2, S3 and S4. Hence, we find that

$$
\begin{aligned}
& \widetilde{F}_{n, 1}(y)=(1-C)\left(\alpha_{1} \theta_{1}+\beta_{1}\right)\left(y^{n}+y^{1-n}\right)+C \\
& \widetilde{F}_{n, 2}(y)=(1-C) \theta_{1}\left(y^{n}-y^{2 m-n}\right)+(1-C) y^{m-n}+C \\
& \widetilde{F}_{n, 3}(y)=(1-C)\left(\alpha_{2} \theta_{1}+\beta_{2}\right)\left(y^{n}-y^{2 m-n}\right)+(1-C) y^{m-n}+C \\
& \alpha_{1}=\frac{y^{2 l}-y^{2 m}}{y^{2 l}+y} \\
& \alpha_{2}=\frac{y^{m}}{y^{2 l}+y} \\
& \beta_{1}=\frac{y^{m}}{y^{2 l}+y} \\
& \beta_{2}=\frac{k_{t} y^{m-l}+y^{m-1-L}\left[\left(u-k_{t}\right) y-u\right]}{y^{L}\left(k_{t}-u+u y\right)-y^{2 m-L-1}\left[\left(k_{t}-u\right) y+u\right]} \\
& \theta_{1}=\left[-y^{m-l}\left(k_{t}+u y\right)+u \beta_{1}\left(y^{l-1}+y^{2-l}\right)+k_{t} \beta_{2}\left(y^{L}-y^{2 m-L}\right)+k_{t} y^{m-L}\right] /\left[y^{l-1}\left(k_{t} y+u\right)\right. \\
& \left.-y^{2 m-l}\left(k_{t}+u y\right)-u \alpha_{1}\left(y^{l-1}+y^{2-l}\right)-k_{t} \alpha_{2}\left(y^{L}-y^{2 m-L}\right)\right]
\end{aligned}
$$

Now it is easy to see that all probability densities in Laplace form obey the relation

$$
\widetilde{F}_{n, i}(y)=P s_{n, i}(y)+C\left(1-P s_{n, i}(y)\right),
$$

where $P s_{y, n}(y)$ is the probability density to find the target while sliding and it contains the dependence on the region $i$ and on the site $n$. Finally, the auxiliary function $S(s)$ is obtained 
after we substitute Eqns. S11-S13, rewriting them in the form of Eqn. S19, to Eqn. S5 in Laplace form. Therefore, we define it as

$$
\begin{aligned}
S(s) & =\sum_{n=1}^{L} P s_{n, i}(y) \\
& =\sum_{n=1}^{l} P s_{n, 1}(y)+\sum_{n=l+1}^{m} P s_{n, 2}(y)+\sum_{n=m+1}^{L} P s_{n, 3}(y) \\
& =\frac{N_{1}}{N_{2}}
\end{aligned}
$$

where we have

$$
\begin{gathered}
N_{1}=u y+2 k_{t} y^{2}+u y^{2}+3 k_{t} y^{3}-u y^{3}+k_{t} y^{4}-u y^{4}+k_{t} y^{1+2 l}+k_{t} y^{2+2 l}-2 k_{t} y^{2-l+L} \\
-4 k_{t} y^{3-l+L}-2 k_{t} y^{4-l+L}-2 k_{t} y^{1+l+L}-4 k_{t} y^{2+l+L}-2 k_{t} y^{3+l+L}+k_{t} y^{1+2 L}-u y^{1+2 L} \\
\quad+3 k_{t} y^{2+2 L}-u y^{2+2 L}+2 k_{t} y^{3+2 L}+u y^{3+2 L}+u y^{4+2 L}+k_{t} y^{3-2 l+2 L}+k_{t} y^{4-2 l+2 L},
\end{gathered}
$$

and

$$
\begin{array}{r}
N_{2}=u y+2 k_{t} y^{2}-u y^{2}-k_{t} y^{3}-u y^{3}-k_{t} y^{4}+u y^{4}+k_{t} y^{1+2 l}-k_{t} y^{2+2 l}-k_{t} y^{1+2 L} \\
+u y^{1+2 L}-k_{t} y^{2+2 L}-u y^{2+2 L}+2 k_{t} y^{3+2 L}-u y^{3+2 L}+u y^{4+2 L}-k_{t} y^{3-2 l+2 L} \\
+k_{t} y^{4-2 l+2 L}-k_{t} y^{2+2 L-2 m}+u y^{2+2 L-2 m}+k_{t} y^{3+2 L-2 m}-u y^{3+2 L-2 m}-u y^{4+2 L-2 m} \\
+u y^{5+2 L-2 m}+k_{t} y^{2+2 l+2 L-2 m}-k_{t} y^{3+2 l+2 L-2 m}+u y^{2 m}-u y^{1+2 m}+k_{t} y^{2+2 m} \\
-u y^{2+2 m}-k_{t} y^{3+2 m}+u y^{3+2 m}-k_{t} y^{2-2 l+2 m}+k_{t} y^{3-2 l+2 m} .
\end{array}
$$

\section{Target located before the transfer site, $m \leq l$}

Similar to the previous case, we divide the DNA strand into 3 regions where the corresponding first-passage functions (in the Laplace form) can be written as:

- $\widetilde{F}_{n, 1}(y)$, for $1 \leq n \leq m$; 
- $\widetilde{F}_{n, 2}(y)$, for $m \leq n \leq l$; and

- $\widetilde{F}_{n, 3}(y)$, for $l \leq n \leq L$.

Using the same methodology from the previous section we are able to find that

$$
\begin{array}{r}
\widetilde{F}_{n, 1}(y)=\frac{(1-C)\left(y^{n}+y^{1-n}\right)}{y^{m}+y^{1-m}}+C \\
\widetilde{F}_{n, 2}(y)=(1-C) \theta_{2}\left(y^{n}-y^{2 m-n}\right)+(1-C) y^{m-n}+C \\
\widetilde{F}_{n, 3}(y)=(1-C)\left(\alpha_{3} \theta_{2}+\beta_{3}\right)\left(y^{n}+\phi y^{-n}\right)+C
\end{array}
$$

with

$$
\begin{aligned}
\phi & =\frac{y^{L}\left(k_{t}-u+u y\right)-k_{t} y^{l}}{-y^{-L-1}\left[\left(k_{t}-u\right) y+u\right]+k_{t} y^{-l}}, \\
\alpha_{3} & =\frac{y^{2 l}-y^{2 m}}{y^{2 l}+\phi}, \\
\beta_{3} & =\frac{y^{m}}{y^{2 l}+\phi}, \\
\theta_{2} & =\left[\beta_{3}\left\{u\left(y^{l+1}+\phi y^{-l-1}\right)+k_{t}\left(y^{L}+\phi y^{-L}\right)\right\}-y^{m-l-1}\left(k_{t} y-u\right)\right] / \\
& \quad\left[y^{l}\left(k_{t}+u y\right)-y^{2 m-l-1}\left(k_{t} y+u\right)+\alpha_{3}\left\{u\left(y^{l+1}+\phi y^{-l-1}\right)+k_{t}\left(y^{L}+\phi y^{-L}\right)\right\}\right]
\end{aligned}
$$

Finally, the auxiliary function for this case is

$$
\begin{aligned}
S(s) & =\sum_{n=1}^{L} P s_{n, i}(y) \\
& =\sum_{n=1}^{m} P s_{n, 1}(y)+\sum_{n=m+1}^{l} P s_{n, 2}(y)+\sum_{n=l+1}^{L} P s_{n, 3}(y) \\
& =\frac{N_{3}}{N_{4}},
\end{aligned}
$$


where

$$
\begin{gathered}
N_{3}=u y+2 k_{t} y^{2}+u y^{2}+3 k_{t} y^{3}-u y^{3}+k_{t} y^{4}-u y^{4}+k_{t} y^{1+2 l}+k_{t} y^{2+2 l}-2 k_{t} y^{2-l+L} \\
-4 k_{t} y^{3-l+L}-2 k_{t} y^{4-l+L}-2 k_{t} y^{1+l+L}-4 k_{t} y^{2+l+L}-2 k_{t} y^{3+l+L}+k_{t} y^{1+2 L}-u y^{1+2 L} \\
\quad+3 k_{t} y^{2+2 L}-u y^{2+2 L}+2 k_{t} y^{3+2 L}+u y^{3+2 L}+u y^{4+2 L}+k_{t} y^{3-2 l+2 L}+k_{t} y^{4-2 l+2 L},
\end{gathered}
$$

and

$$
\begin{array}{r}
N_{4}=u y+2 k_{t} y^{2}-u y^{2}-k_{t} y^{3}-u y^{3}-k_{t} y^{4}+u y^{4}-k_{t} y^{1+2 l}+k_{t} y^{2+2 l}-2 k_{t} y^{2-l+L} \\
+2 k_{t} y^{4-l+L}+2 k_{t} y^{1+l+L}-2 k_{t} y^{3+l+L}-k_{t} y^{1+2 L}+u y^{1+2 L}-k_{t} y^{2+2 L}-u y^{2+2 L}+2 k_{t} y^{3+2 L} \\
-u y^{3+2 L}+u y^{4+2 L}+k_{t} y^{3-2 l+2 L}-k_{t} y^{4-2 l+2 L}-k_{t} y^{2+2 l-2 m}+k_{t} y^{3+2 l-2 m}+2 k_{t} y^{2+l+L-2 m} \\
-2 k_{t} y^{4+l+L-2 m}-k_{t} y^{2+2 L-2 m}+u y^{2+2 L-2 m}-k_{t} y^{3+2 L-2 m}-u y^{3+2 L-2 m}+2 k_{t} y^{4+2 L-2 m} \\
-u y^{4+2 L-2 m}+u y^{5+2 L-2 m}+u y^{2 m}+2 k_{t} y^{1+2 m}-u y^{1+2 m}-k_{t} y^{2+2 m}-u y^{2+2 m}-k_{t} y^{3+2 m} \\
+u y^{3+2 m}-2 k_{t} y^{1-l+L+2 m}+2 k_{t} y^{3-l+L+2 m}+k_{t} y^{2-2 l+2 L+2 m}-k_{t} y^{3-2 l+2 L+2 m} .
\end{array}
$$

